\title{
Conclusion: Cognitive Webs
}

What would we be without our cognitive webs? Like the spider, we create, maintain and manipulate our cognitive webs. Unlike the spider, we also share these webs and learn how to fine-tune them in all sorts of ways. There are webs that are primarily iconic and linguistic ones that are primarily symbolic, and although the webs help us achieve cognitive ends, there are many different ends and many different ways in which the webs allow us to achieve them.

"What do you think about humans and their cognitive webs?" Having read this book, I'd like to hear your answer. 